to persistant dyspnea and fever, a new chest CT was performed and revealed similar image findings but some of the irregular nodular condensations showed a reversed halo sign and had a migrating character. Considering this typical image findings, the absence of causative infectious agents (excluded by bronchoalveaolar lavage performed after delivery) and the absence of response to antibiotics, we concluded with a diagnosis of OP secondary to RA without performing lung biopsy samples. A treatment with $48 \mathrm{mg}$ of methylprednisolone (and $100 \mathrm{mg}$ of azathioprine a few weeks later) was initiated. One month later, she showed a spectacular improvement in her clinical condition. A new chest CT highlighted disappearance of the majority of the ground glass areas and of all the condensations previously described. Methylprednisolone was then progressively tapered.

Conclusion

OP is a common pulmonary complication that can develop in RA. However, despite ou research, we have not found any other clinical case describing this disorder during pregnancy with RA. Since OP is a non-specific inflammatory response to an aggression of the organism, could we consider that the pregnancy is a state of aggression capable of causing such a response?

In addition, this clinical case illustrates the diagnostic challenge of this pathology during the Covid-19 pandemic.

Disclosure of Interests: None declared.

DOI: 10.1136/annrheumdis-2021-eular.49

\section{AB0652 1 FERRITIN IS ASSOCIATED WITH THE SEVERITY OF LUNG INVOLVEMENT BUT NOT WITH WORSE PROGNOSIS IN PATIENTS WITH COVID-19: DATA FROM TWO ITALIAN COVID-19 UNITS}

F. Carubbi ${ }^{1}$, A. Alunno ${ }^{2}$, L. Salvati ${ }^{3}$, C. Ferri ${ }^{1}$, D. Grassi ${ }^{1} .{ }^{1}$ Internal Medicine and Nephrology Unit, University of L'Aquila, Department of Life, Health and Environmental Sciences, L'Aquila, Italy; ${ }^{2}$ Rheumatology Unit, University of Perugia, Department of Medicine, Perugia, Italy; ${ }^{3}$ Infectious Diseases Unit, Ospedale SS Filippo e Nicola ASL 1 Avezzano-Sulmona-L'Aquila, Department of Medicine, Avezzano, Italy

Background: The coronavirus 2019 disease (COVID-19) is characterised by a heterogeneous clinical presentation and a wide range of imaging findings, depending on disease severity and time course. The pathophysiology is complex, involving immune and hematologic systems, epithelial cells and vascular system and to date reliable biomarkers aimed at stratifying patients and predicting worse outcomes have not been identified

Objectives: The aim of this study was to describe clinical, serological and CT imaging features of a cohort of patients with COVID-19 pneumonia and identify possible relationships between the variables and disease outcomes (admission to intensive care unit (ICU) and/or death).

Methods: We evaluated hospitalized patients with proven SARS-CoV-2 infection, clinical signs of COVID-19 and computed tomography (CT) scan-proven pulmonary involvement. Clinical and serological records of patients admitted to two COVID-19 Units in Italy with proven SARS-CoV-2 pulmonary involvement investigated with CT scan, assessed at the time of admission to the hospital, were retrospectively collected.

Results: Sixty-one patients (22 females and 39 males) of median age 65 years were enrolled. Fifty-six patients were discharged while death occurred in 5 patients. None of the lung abnormalities detected by CT was different between discharged and deceased patients. No differences were observed in the features and extent of pulmonary involvement according to age and gender. Logistic regression analysis with age and gender as covariates demonstrated that ferritin levels over the $25^{\text {th }}$ percentile were associated with the involvement of all 5 pulmonary lobes $(\mathrm{OR}=14.5,95 \% \mathrm{Cl}=2.3-90.9, \mathrm{p}=0.004)$, the presence of septal thickening (OR=8.2, $95 \% \mathrm{Cl}=1.6-40.9, \mathrm{p}=0.011)$ and the presence of mediastinal lymph node enlargement $(\mathrm{OR}=12.0,95 \% \mathrm{Cl}=1.1-127.5, \mathrm{p}=0.039)$ independently of age and gender.

Conclusion: We demonstrated that ferritin levels over the $25^{\text {th }}$ percentile are associated with a more severe pulmonary involvement, independently of age and gender, but not with disease outcomes (admission to ICU/death). The identification of reliable biomarkers in patients with COVID-19 may help guiding clinical decision, tailoring therapeutic approaches and ultimately improving the care and prognosis of patients with this disease.

Disclosure of Interests: None declared.

DOI: 10.1136/annrheumdis-2021-eular.6

\section{AB0653 COURSE OF COVID-19 INFECTION IN A SERIES OF PATIENTS WITH SYSTEMIC LUPUS ERYTHEMATOSUS}

C. Cetin $^{1}$, N. Aliyeva ${ }^{1}$, Y. Yalçınkaya ${ }^{1}$, A. Gül ${ }^{1}$, M. Inanc ${ }^{1}$, B. Artim-Esen ${ }^{1}$

${ }^{1}$ Istanbul Faculty of Medicine, Rheumatology, İstanbul, Turkey
Background: Infection is a remarkable cause of morbidity and mortality in patients with SLE.

Objectives: We aimed to determine the clinical course of COVID-19 infection in our patients with SLE and the factors affecting this course

Methods: SLE patients (2012 SLICC criteria) diagnosed with COVID-19 infection by a positive PCR test and/or typical findings of lung involvement in CT (computed tomography) imaging were included. Data regarding cumulative clinical and laboratory characteristics, histopathology results, autoantibody profiles, immunsuppressives and damage (SLICC damage index/SDI)) were retrieved from the existing database and revised. SLE Disease Activity Index (SLEDAI-2K) was determined at the time of infection.

Results: Sixteen SLE patients with COVID-19 infection were identified. Most $(87.5 \%)$ of these patients were female. Seventy \% $(n=11)$ had lupus nephritis. Twenty-five \% had thrombotic antiphospholipid syndrome.

PCR was positive in $70 \%(n=11)$ of the patients. Pulmonary parenchymal findings compatible with COVID-19 were observed in $56 \%(n=9)$ of those patients Regarding complaints upon admission, $50 \%(n=8)$ had fever, $44 \%(n=7)$ cough $44 \%(n=7)$ dyspnea, 19\% $(n=3)$ myalgia, 12.5\% $(n=2)$ headache, $12.5 \%(n=2)$ nausea /vomiting, $6 \%(n=1)$ diarrhea, and $6 \%(n=1)$ had anosmia. Eight patients were hospitalized. Six of these patients needed oxygen therapy via nasal can nula. None needed a follow-up in the intensive care unit. The mean hospitalization duration was $14 \pm 5$ (8-25) days.

Regarding disease activity at the time of infection, 9 had inactive disease with a SLEDAI-2K score of 0 whilst in 5 patients SLEDA-2K score was $\geq 4$. The mean SLEDAI-2K score at the time of infection was $1.7 \pm 2.3(0-6)$. System/ organwise, 1 patient with chronic thrombocytopenia presented with a worsening platelet count accompanied by serologic activity. This patient was a non-adherent to treatment who had stopped taking mycophenolic acid months before COVID19. Three patients 2 of whom had proliferative nephritis experienced nephritic flares. 1 patient who had a history of cutaneous lupus and was in remission presented with oral ulcer, leukopenia and hypocomplementemia during infection. Of 16 patients, 7 had system damage at the time of infection. The mean SDI score of the patients was $1.4 \pm 1.8$. Comparison of patients with and without damage revealed no significant differences in disease activity, symptoms associated with COVID, in the need for hospitalization, hospitalization duration, and the requirement for oxygen therapy. However,CT findings compatible with COVID19, were more common in patients with damage ( $87 \%$ vs. $33 \%, p=0.04)$ and their mean CRP levels were higher at diagnosis $(65 \pm 47$ vs. $22 \pm 48 \mathrm{mg} / \mathrm{l} ; \mathrm{p}=0.032$ )

All patients received similar treatment for COVID-19 except active patients who required high dose steroids ( 2 with active renal, 1 with thrombocytopenia and 1 with oral ulcer, leukopenia and hypocomplementemia). The patient with thrombocytopenia also received intravenous immunoglobulin and 1 with cutaneous active disease received tocilizumab as she developed macrophage activation syndrome. Six patients (37.5\%) had received rituximab (RTX) in the last 6 months before COVID. No significant difference, in terms of hospitalization and need for oxygen therapy due to COVID19 was found between patients who had received RTX vs who had not. No hypogammaglobulinemia was detected in patients who received RTX despite lower levels of IgG (998 \pm 184 vs 1481 $51 \mathrm{mg} / \mathrm{dl}, \mathrm{p}=0.02$ )

Conclusion: Although half of the patients in our series of COVID19 infected SLE patients required hospitalization, there were no mortalities. More patients with damage (none pulmonary) displayed CT findings compatible with COVID19 and further follow up will reveal whether they will suffer from fibrotic lung disease. Patients can experience disease flares during COVID. But it is also important to consider that some manifestations such as thrombocytopenia may also be a sign of severe infection. Immunosupressive agents may not have a negative impact on the course of infection.

*the first two authors contributed equally

Disclosure of Interests: None declared.

DOI: 10.1136/annrheumdis-2021-eular.368

AB0654

TOCILIZUMAB IS PROTECTIVE AGAINST SARS-COV2 INFECTION OR NOT?

F. Fayed ${ }^{1}$, E. Abdelkarim ${ }^{1} .{ }^{1}$ Alexandria University Students ' Hospital, Rheumatology and Immunology Department, Alexandria, Egypt

Background: SARS-COV2 (Covid19) pandemic has a scaring burden on hospitals and health care providers all over the world. Unfortunately, after one year of pandemic, millions of persons are infected either mild symptoms or critical ones and unexplained deaths. Till now, No effective treatment or vaccination. Researches showed that Covid 19 associated with elevated interleukin-6. Subsequently, Studies reported that tocilizumab (IL-6 antagonist) may be effective in critical patients even though other were shown that it is not associated with reduction in the mortality. ${ }^{(1,2)}$ Therefore the question is that patients with rheumatic diseases who are already receiving tocilizumab having a primary 